\title{
Localisation of macrophage lysosomal enzyme in experimental toxoplasma retinitis
}

\author{
MARC O. YOSHIZUMI \\ From the Neurology Research Laboratory, Veteran's Administration Hospital and The University of \\ California, San Francisco, California
}

SUMMARY Rabbit retinal tissue experimentally infected with Toxoplasma gondii was processed for the lysosomal enzyme aryl sulphatase. Abundant lysosomal activity was found in lysosomal bodies of the infected macrophages. There appeared to be a lack of fusion of the lysosomal bodies with the phagosomes containing the organisms. Examination of the majority of macrophage vacuoles containing trophozoites failed to show consistently lead sulphide deposition for aryl sulphatase activity. By light microscopy $83 \%$ of 115 macrophage vacuoles containing the trophozoites of $T$. gondii showed an absence of lysosomal enzyme activity; $7 \%$ of the vacuoles containing the trophozoites were found to contain lysosomal enzyme activity. In the remaining $10 \%$ of the vacuoles containing the trophozoites of $T$. gondii the presence or absence of lysosomal enzyme activity could not be determined with certainty. The frequent absence of lysosomal enzyme activity within the phagosomes containing $T$. gondii organisms may be related to the parasite's ability to multiply and encyst in an intracellular locus. The abundant lysosomal enzyme activity in the lysosomal bodies within the cytoplasm of the infected macrophages may contribute to the cellular destruction of surrounding tissues when infected macrophages burst open owing to proliferation of the trophozoites.

Toxoplasma gondii is one of the commonest causes of retinitis (Jacobs et al., 1956). Toxoplasma has the ability to survive and reproduce by endogeny (Hoare, 1972) in cytoplasmic vacuoles of cells, especially macrophages. It has been speculated that the inability of lysosomes to fuse with the macrophage phagosomes permitted the parasite to resist destruction by hydrolytic enzymes (Jones and Hirsch, 1972). Under these conditions rapid proliferation of organisms ruptured the infected cells.

In previous studies performed in vitro-i.e., tissue culture-the macrophage was precluded from interacting with normal tissue and serum elements such as lymphocytes, plasma cells, and complement which act in vivo to mediate cellular immunity and enhance macrophage phagocytosis. In this in-vivo study rabbit retinal tissue experimentally infected with $T$. gondii was processed for the lysosomal enzyme aryl sulphatase in an attempt to evaluate macrophage lysosomal enzyme activity and localisation during acute retinal infection and tissue destruction.

Address for reprints: Dr Marc O. Yoshizumi, Department of Ophthalmology, Harvard Medical School, Massachusetts Eye and Ear Infirmary, Boston, Massachusetts 02114, USA

\section{Methods and materials}

A $0.05 \mathrm{ml}$ suspension of $\mathrm{RH}$ strain $T$. gondii containing approximately $10^{6}$ free-swimming parasites per $\mathrm{ml}$ in Hanks solution, prepared by the method of Nozik and O'Connor (1965), was injected intravitreally at the equatorial region of the proptosed right globe of white New Zealand rabbits with a No. 27 gauge needle attached to a $1 \mathrm{ml}$ tuberculin syringe.

The rabbits were killed after 5 days, immediately enucleated, and the infected globes immersed in a solution of $1.5 \%$ glutaraldehyde in a $0.1 \mathrm{M}$ cacodylate buffer with $1 \%$ sucrose and $1 \% \mathrm{CaCl}_{2}$. The globes were cut in half at the equator and the vitreous was removed. The posterior retina containing most of the toxoplasma lesions was cut into $1-\mathrm{cm}$ squares on a dental wax slab with a double-edge razor blade. The retina and choroid were dissected from the sclera with a small spatula and surgical blade under direct visualisation with a dissecting microscope. The dissected retina and choroid were futher divided into 3 to $5-\mathrm{mm}$ squares. The specimens were constantly immersed in $1.5 \%$ glutaraldehyde in a cacodylate buffer during the dissecting procedure. 
The tissue specimens were processed for the lysosomal enzyme, aryl sulphatase, according to the method of Goldfischer (1962) and subsequently treated with $2 \%$ ammonium sulphide (Holtzman and Dominitz, 1968). Controls consisted of tissue processed in a substrate-free medium.

On completion of incubation at $37^{\circ} \mathrm{C}$ for 1 hour in the enzyme substrate for aryl sulphatase the tissue was further fixed in a solution of $3 \%$ glutaraldehyde and $1 \%$ paraformaldehyde with $1 \%$ sucrose and $1 \% \mathrm{CaCl}_{2}$ for 1 hour.

After fixation all tissue specimens were stained en bloc with $1 \% \mathrm{OsO}_{4}$ in barbital-acetate buffer at pH 7.6 with $5 \%$ sucrose for 1 hour followed by $0.5 \%$ uranyl acetate in barbital-acetate buffer at pH 6.0 with $5 \%$ sucrose for 1 hour. The stained tissue was dehydrated in increasing concentrations of ethanol followed by propylene oxide and flat embedded in Araldite.

Thick sections for light microscopy were cut on glass knives and stained with Paragon* multiple stain.

Ultrathin sections were cut with a diamond knife,

*Paragon C. \& C. Co. Inc., 190 Willow Avenue, Bronx, New York 10454, USA

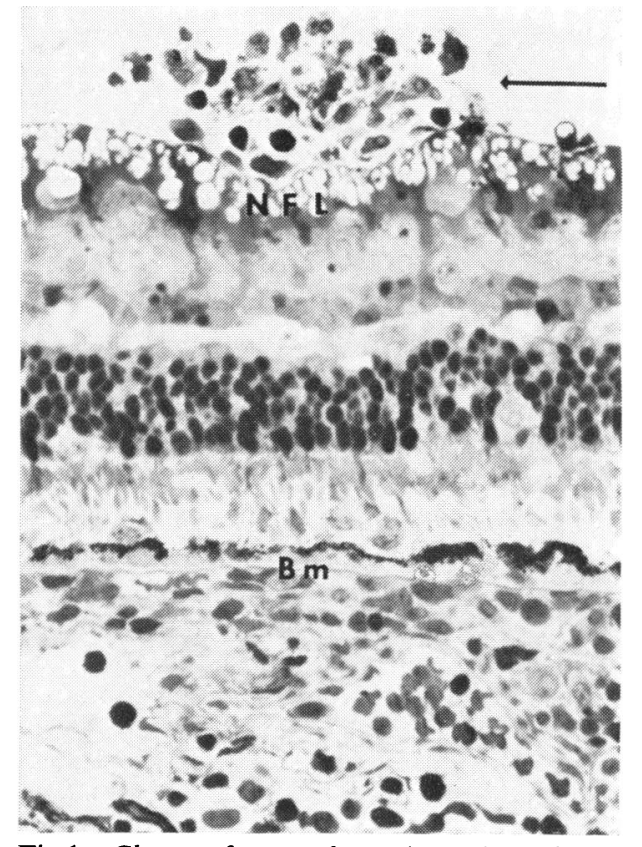

Fig.1 Cluster of macrophages (arrow) on the internal limiting membrane of the retina. The nerve fibre layer (NFL) appears vacuolated. The rest of the retina is intact. Bruch's membrane $(\mathrm{Bm})$ separates the retina above from the choroid below (Araldite thick section with Paragon multiple stain; $\times 400$ )

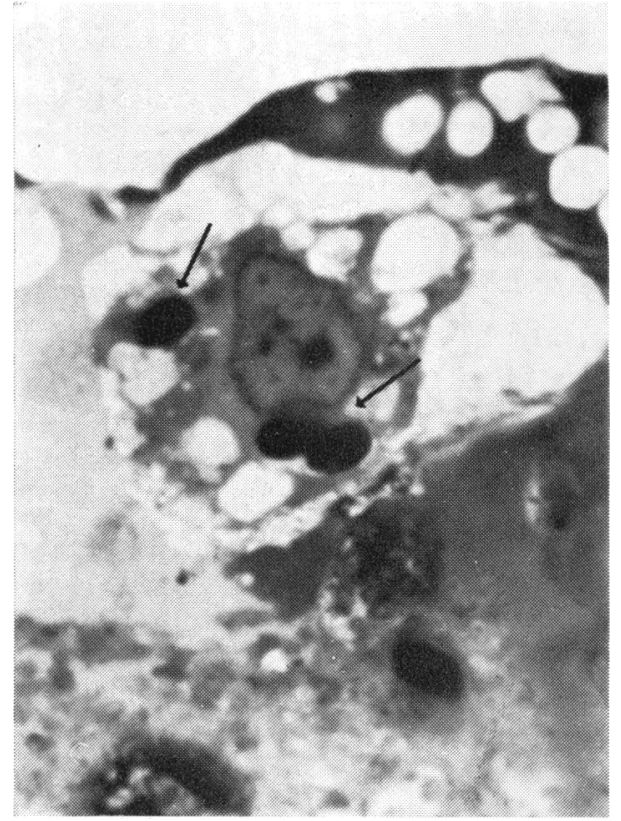

Fig. 2 Higher-power field of the nerve fibre layer with a macrophage containing trophozoites of T. gondii (arrows) within the cytoplasm (Araldite thick section with Paragon multiple stain $; \times 1000$ )

mounted on No. 200 gauge mesh copper grids, and viewed with an electron microscope.

\section{Results}

PHYSICAL SIGNS AND GROSS PATHOLOGY The infected rabbits were found to develop conjunctival injection with a crusty, straw-coloured exudate about 2 to 3 days after the inoculation with $T$. gondii. By the fifth day the rabbits appeared lethargic and somnolent.

Gross examination during the enucleation of the globe revealed no evidence of extension of infection into the sclera or beyond into the orbit. Examination of the retina showed areas of yellowish, pearl-shaped excrescences measuring 1 to $3 \mathrm{~mm}$ in diameter scattered over the posterior retina.

\section{LIGHT MICROSCOPY}

By light microscopy the pearly excrescences were found to be clusters of macrophages over the internal limiting membrane of the retina (Fig. 1). Higherpower examination of these macrophages showed the presence of intracytoplasmic, phagocytised toxoplasma trophozoites (Fig. 2). These organisms were found very commonly in macrophages throughout the retina, involving all layers. Occasionally they were found within the cytoplasm of glial cells. 


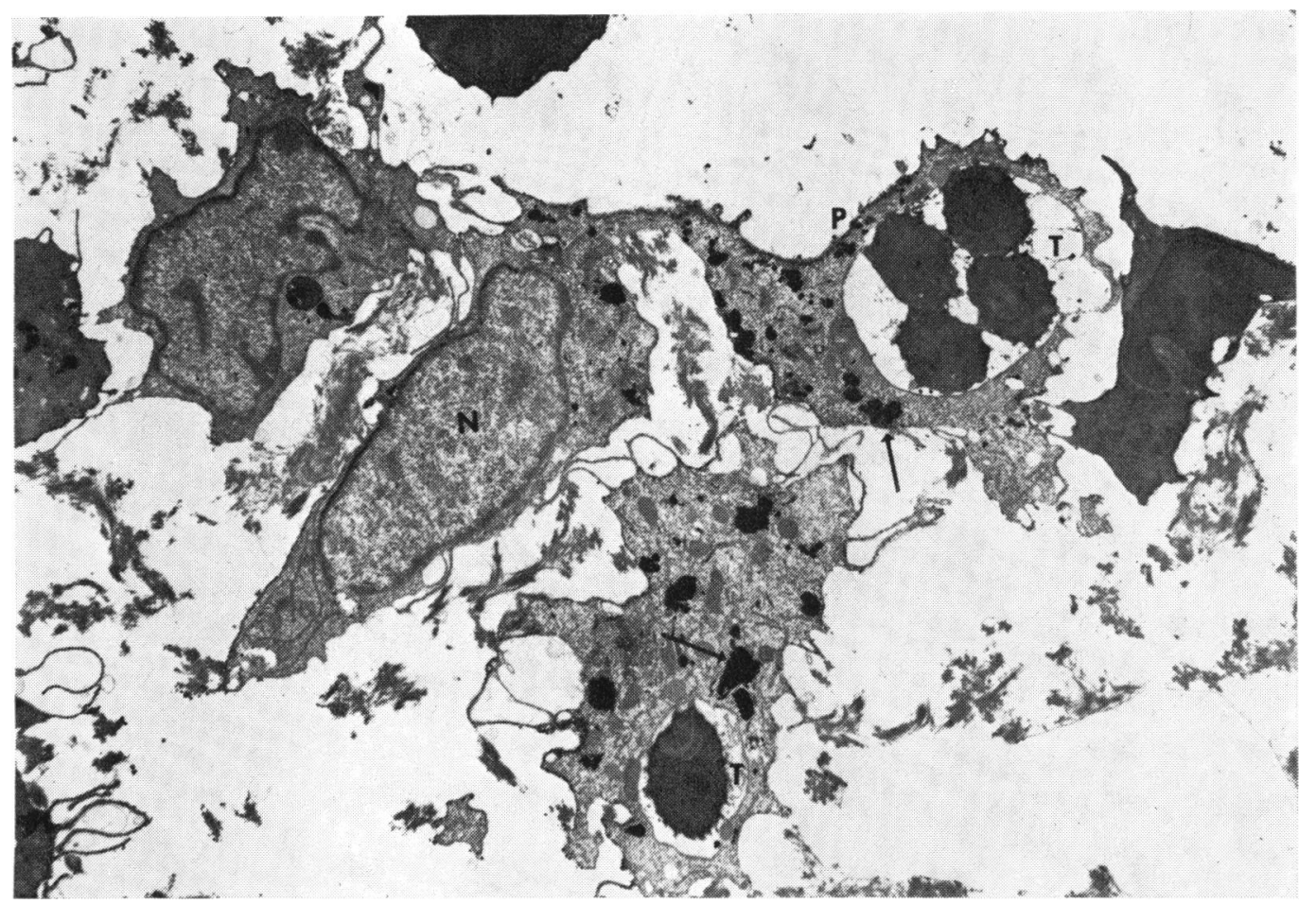

Fig. 3 Macrophage with large nucleus $(N)$ and pseudopodia $(P)$ surrounding an aggregate of trophozoites of T. gondii $(T)$ forming a cytoplasmic vacuole. The lower field shows a portion of a macrophage with a trophozoite $(T)$ in a cytoplasmic vacuole. Dense lead sulphide deposits (arrows) are scattered throughout the cytoplasm of the macrophages. No lead sulphide deposition is noted within the vacuoles surrounding the trophozoites (Aryl sulphatase stained; $\mathrm{OsO}_{4}$ and uranyl acetate; $\times 2000$ )

The lesions of toxoplasma retinitis in the rabbit have been described previously (Yoshizumi, 1976). The optic nerve, ciliary body, anterior chamber, and cornea appeared grossly normal. The vitreous body appeared cloudy.

\section{ELECTRON MICROSCOPY}

Infected retinal tissue stained for aryl sulphatase activity was examined by electron microscopy with special attention to the macrophage vacuoles containing the trophozoites of $T$. gondii. The macrophages were identified by their narrow zone of surface ectoplasm, multiple pseudopodia forming large phagosomal vacuoles, the abundance of mitochondria, and lysosomal bodies in the cytoplasm (Lentz, 1971) (Fig. 3). Heavy deposits of lead sulphide were scattered throughout the cytoplasm of macrophages in lysosomal granules (Fig. 3). However, there was an absence of lead sulphide deposits within the majority of vacuoles containing the trophozoites of $T$. gondii (Fig. 4).

Some dense bodies within the cytoplasm of the trophozoites of $T$. gondii were found to be positive for aryl sulphatase precipitated lead sulphide deposits (Fig. 4).

Control retinal tissue incubated with substrate-free aryl sulphatase showed no specific areas of lead deposition.

Examination of some of the intracytoplasmic vacuoles containing the trophozoites in macrophages showed endoplasmic reticulum and mitochondrial aggregates surrounding the vacuolar membrane (Fig. 5). However, this was not a constant feature around most of the vacuoles containing the parasite.

\section{LIGHT MICROSCOPY OF LEAD SULPHIDE DEPOSITS}

One hundred and fifteen macrophage vacuoles containing the trophozoites of $T$. gondii and stained for aryl sulphatase activity were examined by highpower oil immersion light microscopy. Aryl sulphatase activity was identified by a dense brown lead sulphide precipitate visible by light microscopy.

Ninety-six of 115 or $83 \%$ of the intracytoplasmic macrophage vacuoles containing the trophozoites of 


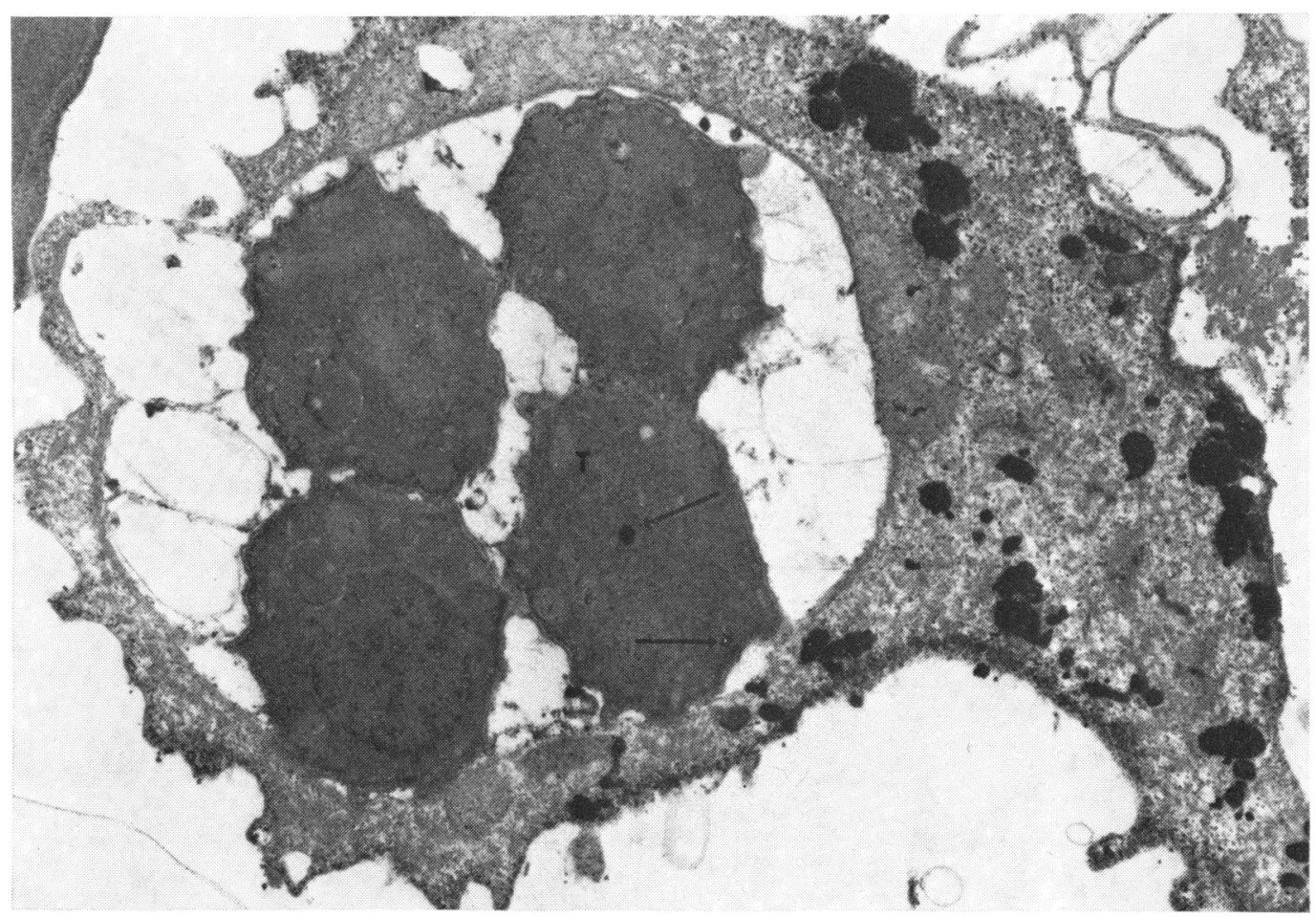

Fig. 4 Higher-power field of Fig. 3. Lead sulphide deposition (arrows) is present within the dense bodies of one of the trophozoites (T) (Aryl sulphatase stained; $\mathrm{OsO}_{4}$ and uranyl acetate; $\times 6000$ )

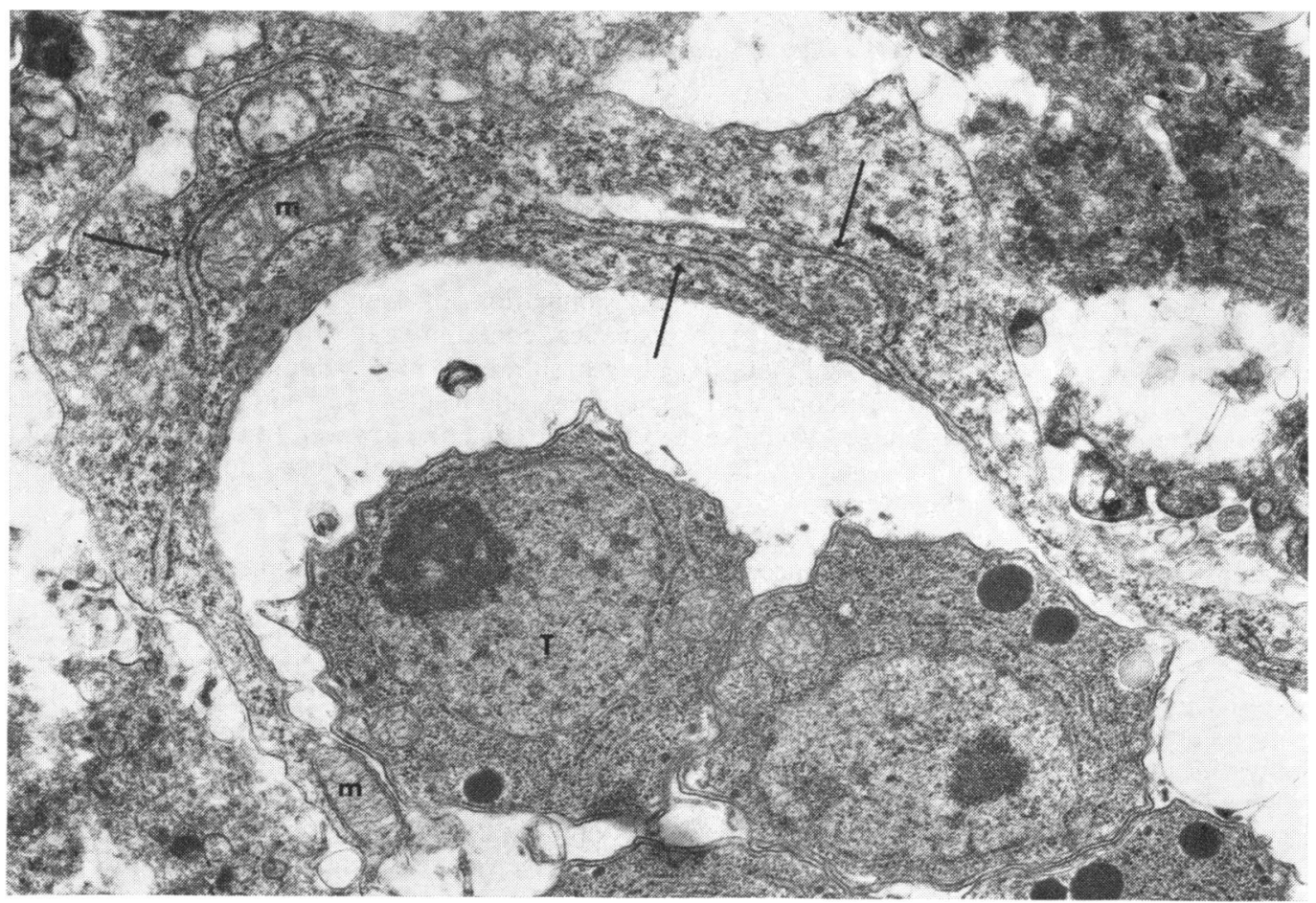

Fig. 5 Trophozoites of $\mathrm{T}$. gondii $(T)$ are within the cytoplasmic vacuole of a macrophage. Surrounding the vacuole membrane are formations of endoplasmic reticulum (arrows) and mitochondria $(\mathrm{m})\left(\mathrm{OsO}_{4}\right.$ and uranyl ace:ate; $\times 7500$ ) 


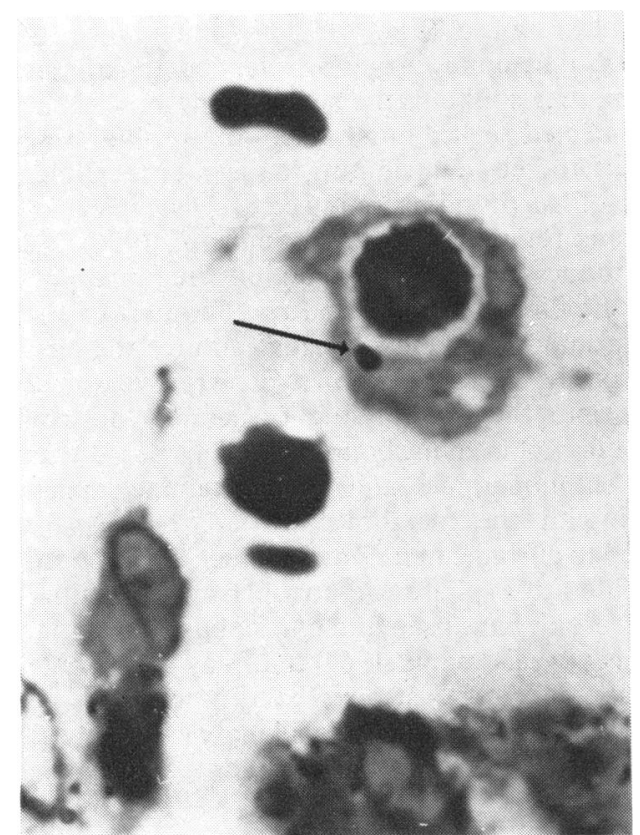

Fig. 6 Light micrograph with a vacuole containing a trophozoite of $\mathrm{T}$. gondii. Lead sulphite deposition is present in the cytoplasm of the macrophage (arrow). No lead deposition is present in the vacuole containing the parasite (Aryl sulphatase stained; $\mathrm{OsO}_{4}$ and uranyl acetate: Araldite thick section stained with Paragon multiple stain; $\times 1500$ )

T. gondii lacked lead sulphide deposits, though deposition was present elsewhere within the cytoplasm of the cell in lysosomal bodies (Fig. 6). Eight of 115 , or approximately $7 \%$, macrophage vacuoles clearly contained both lead sulphide deposits as well as the trophozoites of $T$. gondii and were indicative of aryl sulphatase activity within the macrophage vacuoles containing the parasite. Eleven of 115 , or approximately $10 \%$, macrophage vacuoles containing the trophozoites were equivocal for the presence of lead sulphide deposits and could not be clearly interpreted for aryl sulphatase activity.

\section{Discussion}

\section{VACUOLE FORMATION}

The formation and function of intracytoplasmic vacuoles in cells containing the trophozoites of $T$. gondii has been a point of considerable controversy. Earlier investigators (Lund et al., 1961; Bommer et al., 1969) have maintained that $T$. gondii is an obligate intracellular parasite that actively penetrates the cellular membrane to gain entry into the cell and subsequently, forms intracytoplasmic vacuoles as the site of reproduction. The lysosomes observed in the trophozoites in this study (Fig. 4) have been implicated in facilitating the penetration of the parasite into the host cell (Norrby et al., 1968). In an electron microscopic study Zaman and Colley (1972) observed the anterior end of the $T$. gondii trophozoite penetrating the cell membrane. A membrane was formed around the parasite and the cytoplasm between the newly-formed membrane and the trophozoite dissolved, leaving the organisms in a clear 'parasitophorous' vacuole.

Jones et al. (1972) studied T. gondii trophozoites in HeLa cell, fibroblast, and macrophage tissue cultures at lowered temperatures to retard the entry process of the organisms into the cells and observed no evidence of cellular membrane penetration by the parasite. Instead, the formation of intracytoplasmic vacuoles containing the trophozoites of $T$. gondii appeared to occur through phagocytosis of the organisms by the cells. In the cases of parasitisation of HeLa cells and fibroblasts there was no evidence of destruction of the organisms within the vacuoles but, rather, reproduction of the parasites within the intracytoplasmic locus. In macrophages approximately half of the organisms phagocytised were interpreted to be digested and nonviable. The other half of the phagocytised organisms were interpreted to be viable and multiplied within the intracytoplasmic vacuoles.

\section{LYSOSOMAL ENZYME LOCALISATION}

A subsequent study by Jones and Hirsch (1972) investigated the localisation of lysosomal enzymes in macrophages in tissue culture infected with $T$. gondii and found that $96 \%$ of the vacuoles containing apparently viable organisms showed no evidence of lysosomal enzyme activity. $83 \%$ of the vacuoles containing degenerating trophozoites of $T$. gondii, distinguished on a morphological basis by darker staining and blurring of cellular structure, showed lysosomal enzyme markers.

In this study the lysosomal enzyme aryl sulphatase was examined in retinal tissue from rabbits experimentally infected with $T$. gondii to determine the localisation of the enzyme in vivo rather than in tissue cultured macrophages. It is well established that serum and cell mediated components of the immunological system act in concert with the macrophage in an attempt to destroy invading organisms (Stossel, 1974). Therefore an in-vivo study permits a more physiological evaluation of the fate of the phagocytised parasite in acutely infected retinal tissue rather than in tissue cultured macrophage studies.

$83 \%$ of 115 macrophage vacuoles containing trophozoites of $T$. gondii processed for aryl sulpha- 
tase activity and viewed by light microscopy (Fig. 6) were found to show an absence of lysosomal activity. $7 \%$ of the macrophage vacuoles containing trophozoites were found to contain evidence of aryl sulphatase activity. The absence of lysosomal enzyme markers for aryl sulphatase in the majority of the macrophage vacuoles containing trophozoites was confirmed by electron microscopy. It was not determined whether the trophozoites within the 7\% of macrophage vacuoles with evidence of aryl sulphatase activity were viable or degenerating $T$. gondii organisms. However, the frequent observation of macrophage vacuoles containing the trophozoites of $T$. gondii with absent lysosomal enzyme activity concurs with the findings of tissue culture study by Jones and Hirsch (1972).

Jones and Hirsch (1972) suggest that the viable trophozoites of $T$. gondii may produce a substance which prevents the fusion of lysosomal granules with the membrane of the phagosomes containing the parasite. Evidence for such a substance is still lacking. Another speculation put forward by those investigators is that the endoplasmic reticular and mitrochondrial 'overcoating' around the phagosome containing the trophozoites may block the fusion of lysosomal granules with the phagosome. Evidence for such an 'overcoating' by endoplasmic reticulum and mitochondria was observed in some cells in this study (Fig. 5), but it was not a consistent finding in all cells containing vacuoles with trophozoites and absent lysosomal enzyme (Figs. 3 and 4). Therefore such a mechanism preventing fusion of lysosomal granules with infected phagosomes remains speculative.

The cellular mediated immune response is one of the most significant mechanisms protecting the animal host during infection by the organisms of T. gondii (Frenkel, 1956; O'Connor, 1970). In cellular immunity the macrophage participates in the destruction of organisms by phagolysosomes (Stossel, 1974; World Health Organisation, 1969). The absence of lysosomal activity within the cytoplasmic vacuoles in a majority of the macrophages containing the trophozoites of $T$. gondii in acutely infected retinal tissue may be related to the ability of the parasite to invade, multiply, and encyst in an intracellular locus, and thus maintain a chronic infection characteristic of $T$. gondii retinochoroiditis.

Despite the inability of macrophages effectively to kill ingested trophozoites of $T$. gondii owing to the lack of fusion of the lysosomes with the phagocytic vacuoles the organism appears to elicit a vigorous lysosomal response (Fig. 4). This abundance of hydrolytic lysosomal enzymes, intended to kill the organism, may spill into the surrounding host tissue when infected cells burst due to proliferating organisms; thereby contributing to the intense cellular necrosis of surrounding uninfected cells typical of toxoplasma retinochoroiditis (Yoshizumi, 1976).

Two modes of tissue destruction are apparent. Firstly, the direct invasion and proliferation of the organism in an intracellular locus ruptures the host cell. Secondly, the spillage of hydrolytic lysosomal enzymes into the surrounding tissue may destroy cells that do not even harbour the organisms. Agents such as sulphonamides and pyrimethamine, which interfere with the metabolism of $T$. gondii, are employed to curb the first mode of tissue destruction, the proliferative phase of toxoplasma infection. Corticosteroids have been found to stablise lysosomal membranes (Weissmann, 1964) and may exert some therapeutic effect against the second mode of tissue destruction by reducing the spillage of hydrolytic lysosomal enzymes into the surrounding tissue.

\section{References}

Bommer, W., Heunert, H. H., and Mitthaler, B. (1969). Zeitschrift für Tropenmedizin Parasitologie, 20, 450.

Frenkel, J. K. (1956). Annals of the New York Academy of Sciences, 64, 215.

Goldfischer, S. (1962). Journal of Histochemistry and Cystochemistry, 12, 520.

Hoare, C. A. (1972). Journal of Tropical Medicine and Hygiene, 75, 56.

Holtzman, E., and Dominitz, R. (1968). Journal of Histochemistry and Cytochemistry, 16, 320.

Jacobs, L., Naquin, J. J., Hoover, R., and Woods, A. C. (1956). Bulletin of the Johns Hopkins Hospital, 99, 1.

Jones, T. C., Yeh, S., and Hirsch, J. G. (1972). Journal of Experimental Medicine, 136, 1157.

Jones, T. C., and Hirsch, J. G. (1972). Journal of Experimental Medicine, 136, 1157.

Lentz, T. L. (1971). Cell Fine Structure, p. 66. Saunders: Philadelphia.

Lund, E., Lycke, E., and Sourander, P. (1961). British Journal of Experimental Pathology, 42, 357.

Norrby, R., Lindholm, L., and Lycke, E. (1968). Journal of Bacteriology, 96, 916.

Nozik, R. A., and O'Connor, G. R. (1965). Archives of Ophthalmology, 70, 520.

O'Connor, R. G. (1970). Transactions of the American Ophthalmological Society, 68, 501.

Stossel, T. P. (1974). New England Journal of Medicine, 290, 717, 774 .

Weissman, Q. (1964). Federation Proceedings, 23, 1038.

World Health Organisation (1969). International Archives of Allergy, 36, Suppl. 7.

Yoshizumi, M. O. (1976). Archives of Pathology, 100, 487.

Zaman, V., and Colley, F. C. (1972). Transactions of the Royal Society of Tropical Medicine and Hygiene, 66, 781. 